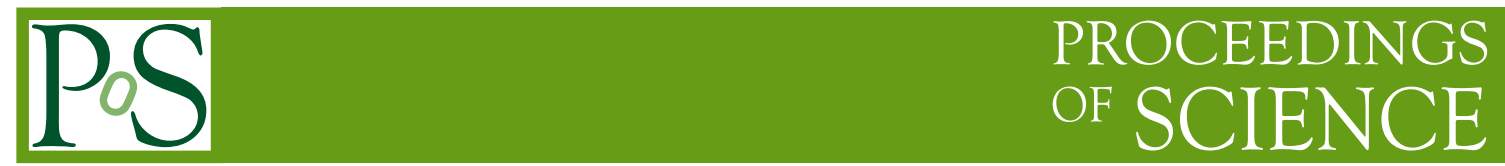

\title{
Long Baseline Neutrinos
}

Takashi Kobayashi*Institute for Particle and Nuclear Studies, High Energy Accelerator Research Organization (KEK)

E-mail: takashi.kobayashi@kek.jp

Status and results of accelerator-based long baseline neutrino oscillation experiments are reviewed. Future prospects are also discussed.

36th International Conference on High Energy Physics

4-11 July 2012

Melbourne, Australia

${ }^{*}$ Speaker. 


\section{Introduction}

In the last decades, great progress in understanding the nature of neutrinos has been made and it is now established that the neutrino have finite mass and "surprisingly" large mixing unlike quarks. However still there are many unanswered questions such as whether the standard $3 \times 3$ mixing matrix picture is correct?, all three flavors participate to the mixing? Why the mixing looks so different from the quarks, CP is violated?, what is the absolute mass?, why so light?, what is the mass spectrum, normal or inverted hierarchy?, etc. The neutrino keeps attracting much attention because unraveling these mysteries could provide breakthrough to understand fundamental questions in particle physics, physics at high energy scale beyond the standard model and origin of matter dominated universe.

Accelerator based long baseline (LBL) neutrino oscillation experiments produce $1 \sim 10 \mathrm{GeV}$ $v_{\mu}$ beam and detect it at several $100 \mathrm{~km}$ from the source. The $v_{\mu}$ beam is produced in the "conventional" method where pions produced by hitting proton beam on a target decay in flight into $v_{\mu}$ as $\pi^{+} \rightarrow v_{\mu}+\mu^{+}$[1]. The physics goals of the LBL experiments have been to understand nature of neutrino, especially mass and flavor mixing structure through the finding and measurements of neutrino oscillation. Neutrino oscillation is a phenomena that the type of neutrino $\left(v_{e}, v_{\mu}\right.$, $v_{\tau}$ ) changes its type during flight if the mass eigenstates of neutrino have different mass eigenvalues each other and the flavor eigenstates are expressed as a linear combinations of more than one mass eigenstates. The typical oscillation length is characterized by $L(\mathrm{~km}) \sim E(\mathrm{GeV}) /\left|\Delta m_{i j}^{2}\right|\left(\mathrm{eV}^{2}\right)$, where $\Delta m_{i j}^{2} \equiv m_{i}^{2}-m_{j}^{2}$ is the mass squared difference of the neutrino mass eigenvalues $m_{i}$. The LBL experiments with $1 \sim 10 \mathrm{GeV}$ and several $100 \mathrm{~km}$ flight distance are optimized for measuring oscillation with $\left|\Delta m^{2}\right| \sim 10^{-2 \sim-3} \mathrm{eV}^{2}$ at which the first evidence of neutrino oscillation was found in atmospheric neutrino observation by Superk-Kamiokande (SK) [2]. The first accelerator-based LBL experiment was the K2K experiment started in 1999 and finished in 2004 in Japan in which the $v_{\mu}$ beam is produced at KEK in Tsukuba-city and detected by Super-Kamiokande at $250 \mathrm{~km} \mathrm{[3].}$ The experiment confirmed the existence of neutrino oscillation by observing disappearance of $v_{\mu}$ after $250 \mathrm{~km}$ flight. The MINOS experiment in US [4] and the OPERA [5] and ICARUS [6] experiments in Europe were planned to further confirm/establish the neutrino oscillation discovered by SK.

Soon after the discovery of neutrino oscillation by SK, the importance of the sub-leading electron neutrino appearance channel was pointed out [7, 8]. In the three flavor mixing picture, the probability of electron neutrino appearance gives a measure of the mixing angle $\theta_{13}$. The existence of electron neutrino appearance at the atmospheric oscillation length means non-zero $\theta_{13}$. Because the $\mathrm{CP}$ violating observable, the phase $\delta$, appears always in the product with $\sin \theta_{12} \sin \theta_{23} \sin \theta_{13}$ and $\theta_{23}$ and $\theta_{12}$ are known to be large, the size of $\theta_{13}$ decides the feasibility of the future CP violation search. With the goal to discover electron neutrino appearance and determine $\theta_{13}$, the T2K experiment [9] in Japan started taking data in 2010 and the NOvA experiment [10, 11, 12] is being constructed to start data taking in 2013.

In these proceedings, the present on-going experiments and future prospects are summarized. 


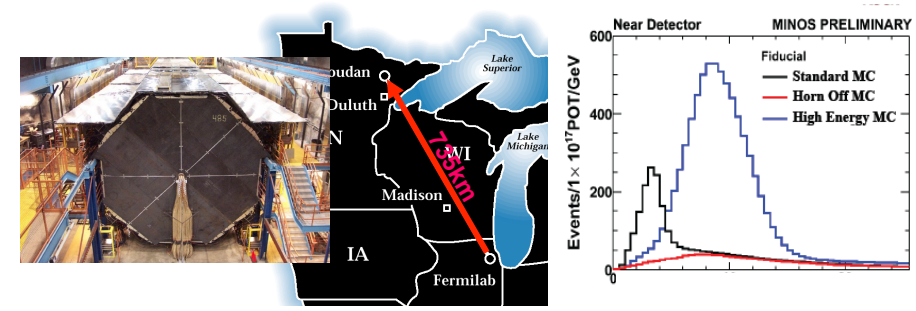

Figure 1: MINOS experiment. Left picture is the far detector in the Soudan mine. The right graphs are expected energy spectra of $v_{\mu} \mathrm{CC}$ interactions (=flux $\times \mathrm{CC}$ cross section). The black histogram is the spectrum in the default MINOS running condition.

\section{Current experiments and their status}

\subsection{MINOS}

MINOS is a long baseline experiment with the precise $v_{\mu}$ disappearance measurements as a main physics goal, in which muon neutrino beam is produced by the $120 \mathrm{GeV}$ proton beam from Main Injector at FNAL and detected by the MINOS detector located in the Soudan mine at $735 \mathrm{~km}$ from FNAL (Fig. 1) [4]. The neutrino beam is a 2-horn focused on-axis wide band beam with the peak energy of around $3 \mathrm{GeV}$ as seen in the expected neutrino spectrum in Fig. 1(right). The beam power of the Main Injector had reached at $350 \mathrm{~kW}$. Magnetized tracking calorimeters are placed at near (980 ton) and far (5,400 ton) site. Physics data taking had been started in May 2005 and finished on Apr. 30, 2012 with the total accumulated POTs of $10.7 \times 10^{20}$ and $3.4 \times 10^{20}$ for $v_{\mu}$ and $\bar{v}_{\mu}$, respectively.

\subsection{CNGS experiments}

CERN neutrino to Gran Sasso (CNGS) is a high energy muon neutrino beam of $\left\langle E_{v}\right\rangle \sim 20 \mathrm{GeV}$ produced at CERN with the SPS $400-\mathrm{GeV}$ proton beam and directed to the detectors placed in Gran Sasso laboratory at $732 \mathrm{~km}$ distance (Fig. 2) [13]. Main goal of the CNGS experiments is to detect $v_{\tau}$ appearance. There are two experiments now taking beam data from CNGS, OPERA [5] and ICARUS [6]. OPERA is an emulsion based detector which identify $\tau$ decay by its decay topology (kink). The detector consists of emulsion based active target part and electronic tracker part. The target part is made of 150,000 $7.5 \times 12.5 \times 10 \mathrm{~cm}^{3}$ emulsion cloud chamber (ECC) blocks, each of which is a stack of 57 emulsion sheets and $561 \mathrm{~mm}$-thick lead sheets, with the total weight of $1.25 \mathrm{kt}$. The OPERA detector started to take neutrino beam data since 2008 and accumulated $1.4 \times 10^{20}$ POT by 2011 and $\sim 1.8 \times 10^{20}$ POT in 2012 .

The other detector is a Liquid Argon TPC detector, ICARUS, which consists of two $3.6 \times$ $3.9 \times 19.6=275 \mathrm{~m}^{3}$ chambers with 476 ton total Liq. Argon mass. It has been taking data since Oct. 2010 and have accumulated $5.8 \times 10^{19}$ POT equivalent data.

\subsection{T2K}

The T2K (Tokai to Kamioka) experiment is a long baseline experiment in Japan with the main goal of discovering $v_{e}$ appearance. The muon neutrino beam is produced by the $30 \mathrm{GeV}$ 


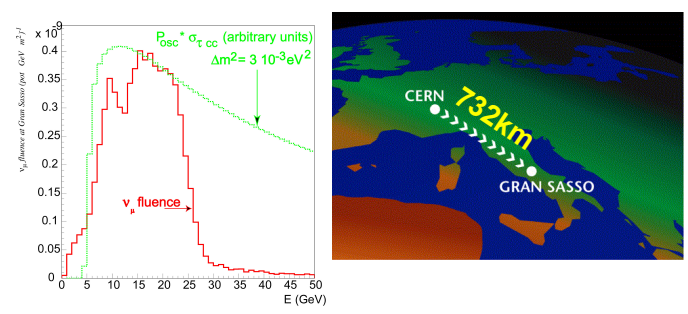

Figure 2: Cern Neutrino beam to Gran Sasso (CNGS). The expected energy spectrum of $v_{\mu}$ flux is plotted in the left panel (red histogram) overlaid with $\left(v_{\tau}\right.$ appearance probability $) \times\left(v_{\tau} \mathrm{CC}\right.$ cross section $)$ as a function of neutrino energy which demonstrate that the $v_{\mu}$ spectrum is optimized to maximize number of $v_{\tau}$ appearance events.
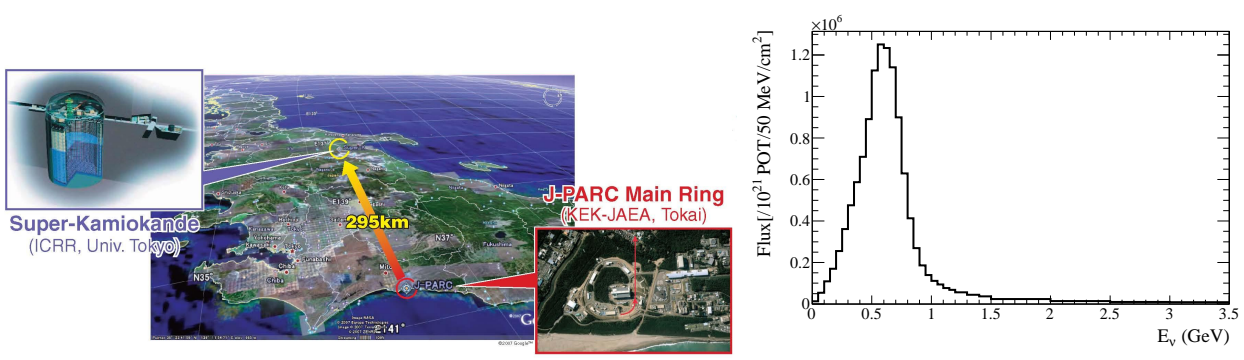

Figure 3: T2K experiment. Expected energy spectrum of $v_{\mu}$ flux at SK is plotted in the right panel.

Main Ring (MR) of J-PARC (Japan Proton Accelerator Research Complex) and detected by the Super-Kamiokande detector at $295 \mathrm{~km}$ from J-PARC. In order to maximize the sensitivity, off-axis scheme is applied for the first time to have intense narrow energy spectrum peaked at the expected oscillation maximum of $0.6 \mathrm{GeV}$. T2K started data taking in Jan. 2010 and achieved $200 \mathrm{~kW}$ stable operation. After one year of recovery work from the big east Japan earthquake on March 11, 2011, the experiment resumed data taking from March 2012 and total accumulated POT reached $3.01 \times 10^{20}$ from the beginning to June 9,2012 .

\section{Important issues in LBL experiments}

The basic method of the analyses in LBL experiments is to compare the observed number of events $N_{f a r}^{o b s}\left(E_{V}\right)$ in a far detector to the expected number of events with oscillation which can be symbolically expressed as

$$
N_{f a r}^{e x p}\left(E_{v}\right)=P_{o s c} \cdot \Phi_{f a r} \cdot \sigma_{f a r} \cdot \varepsilon_{f a r}
$$

in a simplified formula omitting to explicitly write integration of, for example, final state particle phase space, and $P_{o s c}, \Phi_{f a r}, \sigma_{f a r}$ and $\varepsilon_{f a r}$ are oscillation probability, neutrino flux, neutrino interaction cross section and detection efficiency at the far detector. The precision or sensitivity of a LBL experiment is determined by how precisely $N_{\text {far }}^{\text {exp }}$ can be evaluated (and of course the statistical error of $N_{f a r}^{o b s}$ ). 


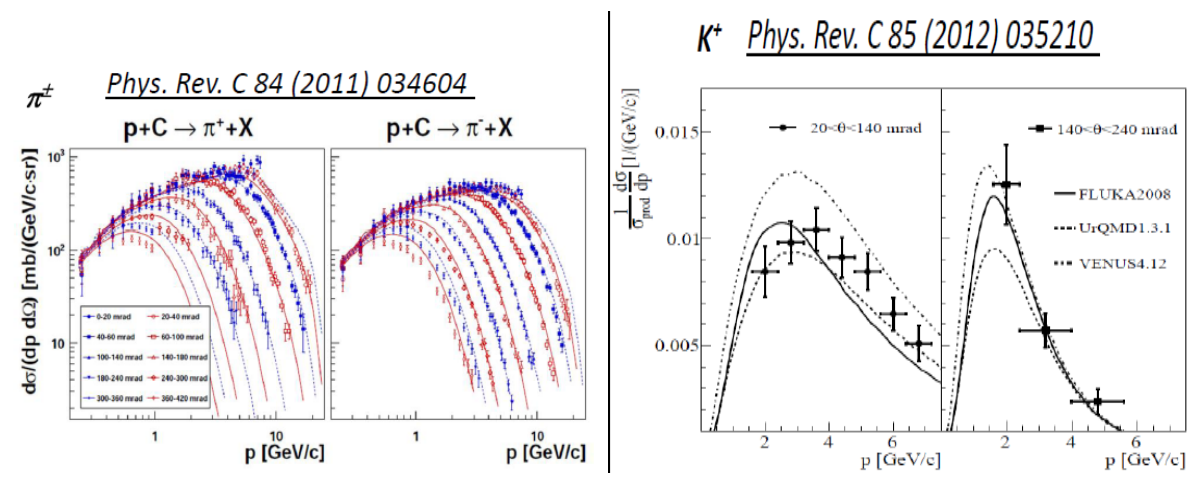

Figure 4: Example of results from hadron production measurements in NA61 for T2K for pion (left) and Kaon (right)

In order to predict $N_{\text {far }}^{e x p}$ precisely, usually it is evaluated by extrapolating the measurement by a near detector $N_{\text {near }}^{\text {obs }}$

$$
N_{\text {far }}^{\text {exp }}\left(E_{V}\right)=N_{\text {near }}^{\text {obs }} \cdot \frac{P_{\text {osc }} \cdot \Phi_{\text {far }} \cdot \sigma_{\text {far }} \cdot \varepsilon_{\text {far }}}{\Phi_{\text {near }} \cdot \sigma_{\text {near }} \cdot \varepsilon_{\text {near }}}
$$

The energy spectra of $v_{\mu}$ flux at near and far site (without oscillation) are not the same due to geometrical difference relative to the neutrino production region and also the cross section and efficiencies are usually different for near and far detector, so this extrapolation does not follow simple $1 / R^{2}$ law. However, by this extrapolation, partial cancellation of systematic errors associated with absolute normalization, energy spectrum prediction, and neutrino interaction cross sections are expected.

As seen in these formula, important elements which determine the precision are

- Beam flux (absolute normalization, spectrum shape and near to far extrapolation)

- Neutrino interaction cross section

- Detection efficiencies of far and near detectors

For the beam flux and cross section, as seen in the following examples, in situ or supporting measurements have been playing critical roles for achieving high precision in LBL experiments.

\subsection{Understanding beam}

The neutrino flux at near and far site and their relation are governed by parent hadron $(\pi / \mathrm{K}$, etc) distributions at production (momentum $p$ and polar angle $\theta$ ) and beam line geometry. Because the geometory is under control, precise knowledge of hadron production is essential to predict neutrino flux and extrapolation from the flux at near site to the one at the far site precisely.

Dedicated measurements of hadron production for LBL experiments have been made in the HARP experiment at CERN-PS for K2K and MiniBooNE experiments [14, 15, 16], MIPP at FNAL for MINOS [17], NA61 at CERN-SPS for T2K and US experiments [16, 18, 19]. Example of such measurements from NA61 are plotted in Fig. 4. These hadron production experiments have been providing critically important data for improving the precision of LBL experiments. 

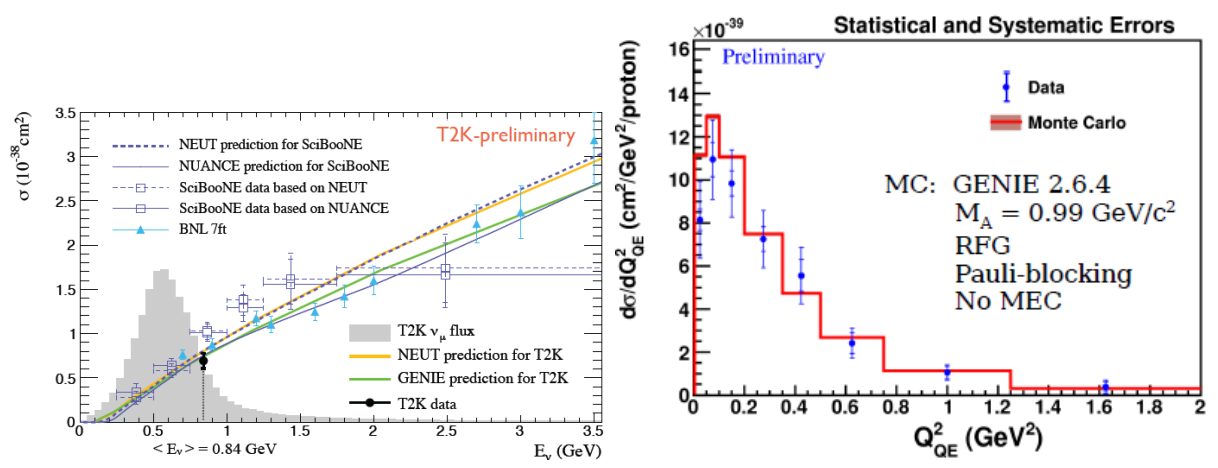

Figure 5: Measurements of neutrino interaction cross sections. Left is the result of inclusive $v_{\mu} \mathrm{CC}$ cross section from T2K [23] and the right is preliminary results of $\bar{v}_{\mu} \mathrm{CCQE}$ cross section measured by the MINERvA experiment[20].

\subsection{Understanding cross sections}

Another important element for the precision LBL experiments is understanding neutrino interaction cross sections. For that purpose, dedicated experiments such as MINERvA [20] and oscillation experiments themselves such as K2K [21, 22], T2K [23, 24] and MiniBooNE [25] have been providing important results [26]. Example of such results are quoted in Fig. 5

\section{Results}

\section{1 $v_{e}$ appearance}

In 2011, T2K observed the first indication of $v_{e}$ appearance in the $v_{\mu}$ beam at $2.5 \sigma$ significance by detecting $6 v_{e}$ event candidates beyond $1.5 \pm 0.3 \mathrm{BG}$ expectation with $1.43 \times 10^{20}$ POT data [27]. At this conference, T2K released the new result of $v_{e}$ appearance with $3.01 \times 10^{20}$ POT data taken nutil June 9th, 2012 [28]. In T2K, signal event of $v_{e}$ appearance should have only one electron-like Čerenkov ring which comes from $v_{e}$ CCqe interaction $v_{e}+n \rightarrow e^{-}+p$. Dominant background sources are (1) intrinsic $v_{e}$ in the beam and (2) $\mathrm{NC} \pi^{0}$ interactions in which one of electromagnetic showers from two decay- $\gamma_{\mathrm{s}}$ is missed. The signal events are selected by requiring 1) $1 \mathrm{e}$-like ring w/ visible energy $\left.\left(E_{v i s}\right)>100 \mathrm{MeV}, 2\right)$ No decay electron detected, 3) Force to find a 2nd ring although standard ring counting found only 1 ring, and reconstructed invariant mass with the original ring and the forced-found 2nd ring be less than $105 \mathrm{MeV}$ to reject remaining $\pi^{0}$, 4) Reconstructed electron neutrino energy $E_{v}^{r e c}<1250 \mathrm{MeV}$. Eventually, 11 signal candidate are found. Their $E_{v}^{\text {rec }}$ distribution is plotted in Fig. 6 together with expected distributions. The expected observation at SK is predicted in principle by extrapolating the neutrino measurement at near site using beam MC simulation validated by the hadron production data actually measured by NA61. In Fig. 6, muon momentum distribution of events selected as $v_{\mu}$ CCqe interactions by the near detector is plotted. Absolute flux and spectrum shape of neutrino beam and neutrino interaction cross sections are tuned to reproduce this observation. The tuned MC prediction reproduce the observation very well as seen in Fig. 6. Expected number of events in SK thus obtained are $3.2 \pm 0.4$ (10.7 \pm 1.1 ) for $\sin ^{2} 2 \theta_{13}=0(0.1)$. The significance of the excess beyond the background expectation is calculated 

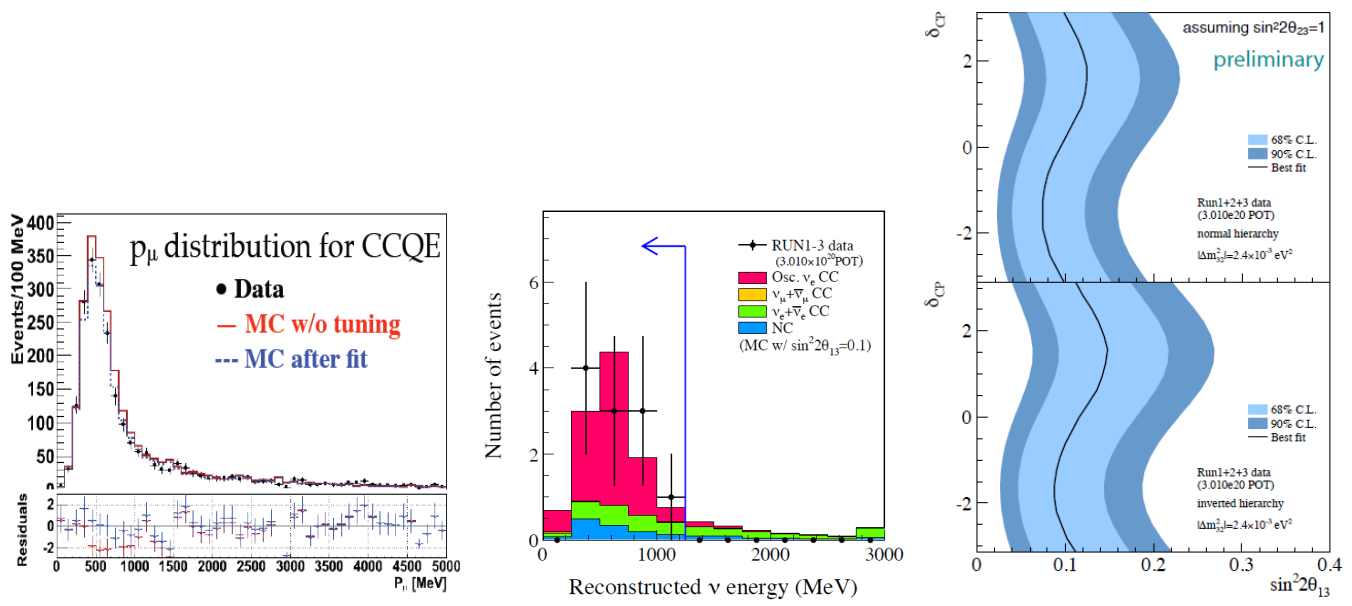

Figure 6: (Left) Muon momentum distribution of $v_{\mu}$ CCQE reactions measured by the T2K near detector. (Center) Reconstructed neutrino energy distribution of $\mathrm{T} 2 \mathrm{~K} v_{e}$ appearance candidate events. (Right) Allowed region of oscillation parameters $\sin ^{2} 2 \theta_{13}$ and $\delta_{C P}$.

to be $3.2 \sigma$ or the probability to observe 11 events with $\theta_{13}=0$ is $0.08 \%$. T2K detected an evidence of $v_{e}$ appearance for the first time which open a possibility to detect $\mathrm{CP}$ violation in the lepton sector.

The MINOS experiment also has been making efforts to search for $v_{e}$ appearance [29, 30]. In order to select electron neutrino candidate events, events are required 1) to be in fiducial volume, 2) to match with the expected beam timing, 3) not associated with tracks longer than 24 planes or a track extending more than 15 planes beyond the end of a reconstructed shower activity to reject events with a muon, 4) to have 5 contiguous planes with an energy deposition at least half that of a minimum ionizing particle, 5) to have energy from 1 to $8 \mathrm{GeV}$. In order to further reduce the background, in the latest analysis, a nearest-neighbor "library event matching" (LEM) technique is used. With an exposure of $10.6 \times 10^{20}$ POT in the neutrino-enhanced beam, MINOS observed 152 electron candidate events in the Far detector while 127.7 (161.4) events are expected assuming $\sin ^{2} 2 \theta_{13}=0\left(\sin ^{2} 2 \theta_{13}=0.1, \delta=0\right.$, normal mass hierarchy). With an exposure of $3.3 \times 10^{20}$ POT in the antineutrino-enhanced beam, MINOS observed 20 signal candidate events while 17.5 (21.4) events are expected in the Far detector assuming $\sin ^{2} 2 \theta_{13}=0\left(\sin ^{2} 2 \theta_{13}=0.1\right.$, $\delta=0$, normal mass hierarchy). The allowed regions as a function of the CP violating phase, $\delta$, and $2 \sin ^{2}\left(2 \theta_{13}\right) \sin ^{2} \theta_{23}$ are shown in Figure 7. For $\delta=0$ and the normal (inverted) mass hierarchy a best fit of $2 \sin ^{2}\left(2 \theta_{13}\right) \sin ^{2} \theta_{23}=0.051$ (0.093) is obtained.

At CNGS, the OPERA experiment searches for the $v_{e}$ appearance although the $L / E$ is not optimized to the atmospheric $\Delta m^{2}$ region of $\sim 3 \times 10^{-3} \mathrm{eV}^{2}$. Events with an electron from the primary vertex are selected [31]. Observed number of events is 19 (4) while expected number of oscillated $v_{e}$ events and beam $v_{e}$ backgrounds are 1.5 (1.1) and 19.2 (3.7) for all neutrino energy region $\left(E_{v}^{r e c}<20 \mathrm{GeV}\right)$. The observations are consistent with background expectation. The distributions of the reconstructed neutrino energy for the observed events and MC predictions are plotted in Fig. 7. The exclusion contour is also plotted in Fig. 7. For $\Delta m^{2} \gtrsim 10^{-2} \mathrm{eV}^{2}$, the mixing angle $\sin ^{2} 2 \theta_{13}$ region greater than a few times $10^{-2}$ is excluded. 

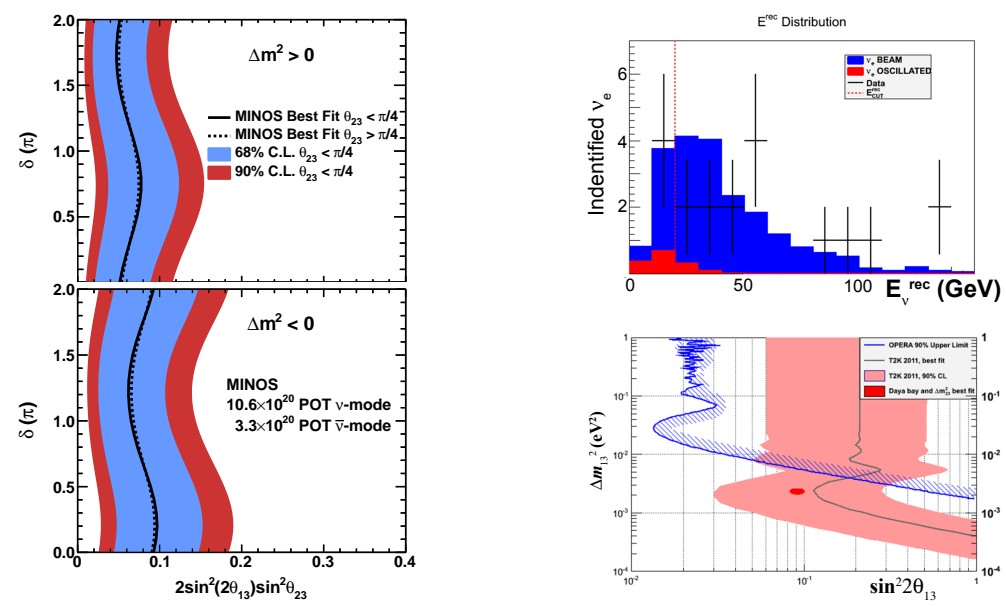

Figure 7: (Left 2 plots)Allowed regions of oscillation parameters $\sin ^{2} 2 \theta_{13}$ and $\delta_{C P}$ by the MINOS appearance search. (Right 2 plots) Results from OPERA $v_{e}$ appearance search. Top plot is the neutrino energy distribution of the detected $v_{e}$ event candidates together with MC expectations of signal (red) and background(blue). Bottom plot is excluded region by OPERA (upper right of the blue curve) with the allowed regions of other experiments.

\section{$4.2 v_{\mu}$ disappearance}

Measurements of $v_{\mu}$ disappearance determine the oscillation parameters $\theta_{23}$ and $\Delta m_{23}^{2}$. In contrast to the atmospheric neutrino measurements, flight distance $L$ is precisely known in LBL experiments. Therefore, precise measurements are possible for the oscillation parameters $\theta_{23}$ and $\Delta m_{23}^{2}$ from the neutrino energy distribution.

MINOS experiment has been providing the most precise measurements of $v_{\mu}$ disappearance beside the Super-Kamiokande atmospheric neutrino measurement. Recently MINOS released new preliminary results using the complete data set $[29,32]$. With the total neutrino-enhanced beam exposure of $10.7 \times 10^{20}$ POT, MINOS observed 2894 fully reconstructed $v_{\mu}$ CC events while 3564 events are predicted assuming no oscillation. Figure 8 shows the $E_{v}$ distribution of the fully reconstructed events where the distortion of the energy spectrum expected by oscillations can be clearly seen. In addition, in the latest analysis, two additional data sets are included: the antineutrinoenhanced beam data $\left(3.36 \times 10^{20} \mathrm{POT}\right)$ and atmospheric (anti-)neutrinos (37.9 kiloton-years). The best fit point with all these sample are $\left|\Delta m^{2}\right|=\left(2.39_{-0.10}^{+0.09}\right) \times 10^{-3} \mathrm{eV}^{2}$ and $\sin ^{2}(2 \theta)=0.96_{-0.04}^{+0.04}$ and allowed region is plotted in Fig. 8.

$\mathrm{T} 2 \mathrm{~K}$ also started to contribute the measurement of $v_{\mu}$ disappearance [33]. Using the data corresponding to $1.43 \times 10^{20}$ POT taken before the long shutdown due to the earthquake, T2K selected $v_{\mu}$ CCqe events by requiring fully-contained in fiducial volume, single muon-like ring with 0 or 1 decay electron associated, $p_{\mu}>200 \mathrm{MeV} / \mathrm{c}$ and found 31 events against an expectation of $104 \pm 14$ (syst) without neutrino oscillations. The observed and expected reconstructed $E_{v}$ distributions are plotted in Fig. 8. The allowed region given by T2K is overlaid in the Fig. 8. Latest preliminary SK results are also drawn in the same figure and all the results from different experiments are consistent with each other. 

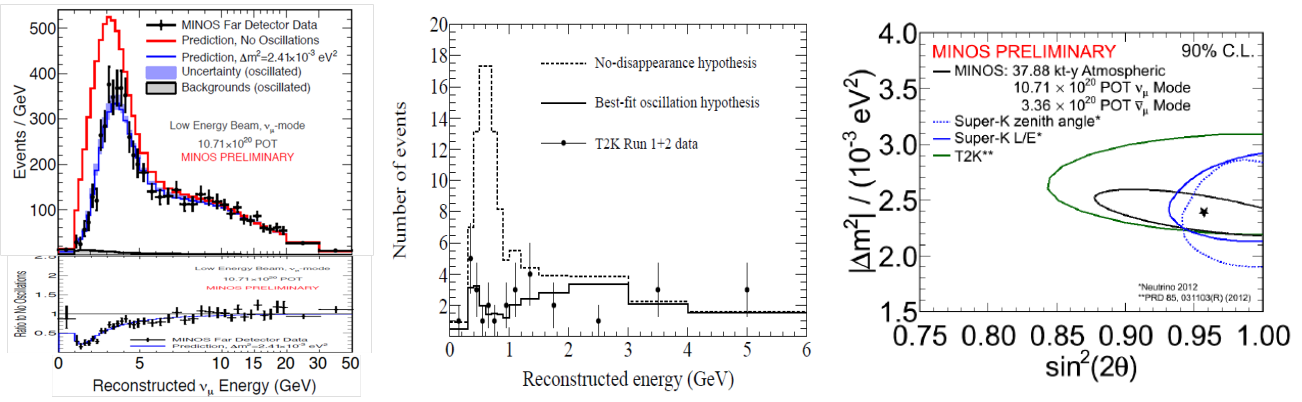

Figure 8: Reconstructed neutrino energy distributions of $v_{\mu}$ events at far detectors in MINOS (Left) and T2K (Center) experiments. Points with error bars are data and the histograms are expected distributions with and without disappearance. (Right) Allowed parameter regions by disappearance measurements by various experiments.

\section{$4.3 v_{\tau}$ appearance search}

The main goal of the OPERA experiment is the $v_{\tau}$ appearance search. The $v_{\tau}$ interactions are identified by decay kink of $\tau$ from CC $v_{\tau}$ interaction with the fine tracking capability of ECC. OPERA reported in 2010 that one $v_{\tau}$ candidate event was found in the data taken in 2008-2009 corresponding to $5.30 \times 10^{19}$ POT. OPERA is analyzing $8.88 \times 10^{19}$ POT data taken in 20102011 and another candidate event is found so far. In total 2 candidate events has been found against expected $v_{\tau}$ appearance and background events are 2.1 and 0.2 [31]. Systematic error on the expectation is being estimated.

Although it is not accelerator based LBL experiment, SK has been searching for $v_{\tau}$ appearance in the $v_{\tau}$ enhanced sample of atmospheric neutrinos. With the 2806 days of data ( $=173 \mathrm{kt} \cdot \mathrm{yrs}$ or 63.1 Mt.days), $v_{\tau}$ signal excess over the background is found to be $170.8 \pm 44.3(\text { stat })_{-15.2}^{+17.8}($ syst $)$ events which is $3.8 \sigma$ evidence of $v_{\tau}$ appearance [34].

\section{Future}

T2K found the first evidence of $v_{e}$ appearance by positively identifying the CC interactions of the appeared flavor. Reactor experiments clearly observed $\bar{v}_{e}$ disappearance with more than $7.7 \sigma$ significance and provide precise measurements of $\theta_{13}$ [35]. Discovery of large $\theta_{13}$ provided great opportunities to understand the unknown nature of neutrinos, such as $\mathrm{CP}$ symmetry and mass hierarchy.

Reactor disappearance measurements primarily depend only on $\theta_{13}$ and $\Delta m_{13}$ while the appearance probability depends not only on them but also on various other parameters including $\delta_{C P}$ and sign of $\Delta m_{23}^{2}$. Threrefore, in the near term future, it is very important to improve the precision of the appearance probability as much as possible by LBL experiments $\mathrm{T} 2 \mathrm{~K}$ and NOvA which could enable to constrain $\delta_{C P}$ and/or mass hierarchy by combining with the precise $\theta_{13}$ measurements by reactor experiments.

The $v_{\mu}$ disappearance is now getting more important. The CP violating term in the $v_{e}$ appearance probability is symmetrically proportional to all mixing angles $\sin \theta_{12}, \sin \theta_{23}$ and $\sin \theta_{13}$. Therefore, in order to constrain $\delta_{C P}$ from precision measurements of the appearance probability, 
high precision of similar level for all three angles are necessary. Thanks to the precision measurements of $\theta_{13}$, now least known mixing angle is $\theta_{23}$ which can be measured by the $v_{\mu}$ disappearance. $\mathrm{T} 2 \mathrm{~K}$ and NOvA experiments are expected to play important role to constrain $\theta_{23}$ through precision measurement of $v_{\mu}$ disappearance for coming years.

For the longer term future, efforts to formulate experiments to attack the $\mathrm{CP}$ violation and mass hierarchy are being made in the world taking into account the new knowledge of the large $\theta_{13}$. In the US, the proposed LBNE project, in which the neutrino beam is produced at FNAL and detected by a 34 kton Liq. Argon TPC in the Homestake mine at 1300km from FNAL, is reconfigured into a phased approach starting with a $10 \mathrm{kt}$ detector on surface first [36]. In Europe, EoI of the LBNO project is submitted to CERN SPSC [37]. The primary option of the project is to produce neutrino beam using new facility at CERN SPS and direct to a detector placed at $2300 \mathrm{~km}$ from CERN in Finland. In the first phase, 20 kton LAr TPC is used to determine the mass hierarchy. In the second phase, possibilities of $100 \mathrm{kton}$ class LAr TPC at $2300 \mathrm{~km}$ and $2 \times 300 \mathrm{kton}$ class Water Čerenkov detector at $130 \mathrm{~km}$ (Frejus) with 4MW beam from high power SPL are being discussed. In Japan, two options for future LBL experiments are being discussed; One is a 1-Mt class Water Čerenkov detector, Hyper-Kamiokande in Kamioka at $\sim 300 \mathrm{~km}$ from J-PARC, $2.5^{\circ}$ off axis [38] and the other is $100 \mathrm{kt}$ class LAr TPC in Okinoshima island at $658 \mathrm{~km}$ from J-PARC, $0.78^{\circ}$ off-axis [39].

\section{Summary}

Present status and results, and future prospects of LBL experiments are reported. The $3.2 \sigma$ evidence of $v_{e}$ appearance is reported by T2K experiment. It opened possibility of CP violation and mass hierarchy determination. MINOS also reported $2 \sigma$ level appearance signal. The OPERA experiment is looking for $v_{\tau}$ appearance using fine tracking detector with emulsion technique. So far $2 v_{\tau}$ candidate events are detected while the expected appearance signal and the background expectation are 2.1 and 0.2, respectively. Although it is not accelerator LBL experiment, the SuperKamiokande observed $v_{\tau}$ appearance at $3.8 \sigma$ significance in the atmospheric neutrino observation. For the $v_{\mu}$ disappearance, $\Delta m_{23}^{2}$ is now most constrained by MINOS as $2.39_{-0.11}^{+0.09} \times 10^{-3} \mathrm{eV}^{2}(4 \%$ meas) and $\theta_{23}$ by SK atm $v$ observation as $\sin ^{2} 2 \theta_{23}>0.93 \sim 0.95$ (90\%CL).

The discovery of finite and large $\theta_{13}$ opened the possibility to find $\mathrm{CP}$ violation and determine mass hierarchy in the future. In the near term, precision measurements of $v_{e}$ appearance and $v_{\mu}$ disappearance in LBL experiments combined with precision $\theta_{13}$ measurement from reactor experiments can start constraining $\mathrm{CP}$ violation and mass hierarchy. Future large scale experiments optimized for CPV and Mass hierarchy are being discussed and proposed in the world.

\section{References}

[1] S. E. Kopp, “Accelerator-based neutrino beams," Phys.Rept. 439 (2007) 101-159.

[2] Super-Kamiokande Collaboration, Y. Fukuda et al., "Evidence for oscillation of atmospheric neutrinos," Phys.Rev.Lett. 81 (1998) 1562-1567.

[3] K2K Collaboration, M. Ahn et al., "Measurement of Neutrino Oscillation by the K2K Experiment," Phys.Rev. D74 (2006) 072003. 
[4] MINOS Collaboration, E. Ables et al., "P-875: A Long baseline neutrino oscillation experiment at Fermilab,” FERMILAB-PROPOSAL-0875, NUMI-L-79.

[5] OPERA Collaboration, M. Guler et al., "OPERA: An appearance experiment to search for $v_{\mu} \rightarrow v_{\tau}$ oscillations in the CNGS beam. Experimental proposal,” CERN-SPSC-2000-028, CERN-SPSC-P-318, LNGS-P25-00.

[6] ICARUS Collaboration, F. Arneodo et al., "The ICARUS experiment, a second-generation proton decay experiment and neutrino observatory at the Gran Sasso Laboratory," arXiv:hep-ex/0103008.

[7] K. Nishikawa Presented at the Europhysics Neutrino Oscillation Workshop (NOW'98), 7-9 September 1998, Amsterdam.

[8] T2K Collaboration, Y. Itow et al., "The JHF-Kamioka neutrino project," arXiv:hep-ex/0106019 [hep-ex].

[9] T2K Collaboration, K. Abe et al., “The T2K Experiment,” Nucl.Instrum.Meth. A659 (2011) 106-135.

[10] D. Ayres et al., "Letter of Intent to build an Off-axis Detector to study numu to nue oscillations with the NuMI Neutrino Beam," arXiv:hep-ex/0210005 [hep-ex] .

[11] NOvA Collaboration, D. Ayres et al., "NOvA: Proposal to build a 30 kiloton off-axis detector to study $v_{\mu} \rightarrow v_{e}$ oscillations in the NuMI beamline," FERMILAB-PROPOSAL-0929, arXiv:hep-ex/0503053 [hep-ex].

[12] NOvA Collaboration, D. Ayres et al., "The NOvA Technical Design Report," FERMILAB-DESIGN-2007-01 (2007) .

[13] G. Acquistapace et al., "The CERN neutrino beam to Gran Sasso (NGS)," CERN-98-02, INFN-AE-98-05, CERN-YELLOW-98-02 (1998) .

[14] HARP Collaboration, M. Catanesi et al., "Measurement of the production cross-section of positive pions in p-Al collisions at 12.9-GeV/c," Nucl.Phys. B732 (2006) 1-45.

[15] HARP Collaboration, M. Catanesi et al., "Measurement of the production cross-section of positive pions in the collision of 8.9-GeV/c protons on beryllium," Eur.Phys.J. C52 (2007) 29-53.

[16] B. A. Popov, "Hadron production experiments," Neutrino Conference Proceedings (2012), arXiv:1212.1030 [hep-ex].

[17] S. Mahajan, A. Kumar, and R. Raja, "Main injector particle production experiment at Fermilab," Pramana 79 (2012) 1243-1246.

[18] NA61/SHINE Collaboration, N. Abgrall et al., "Measurements of Cross Sections and Charged Pion Spectra in Proton-Carbon Interactions at 31 GeV/c," Phys.Rev. C84 (2011) 034604.

[19] NA61/SHINE Collaboration, N. Abgrall et al., "Measurement of Production Properties of Positively Charged Kaons in Proton-Carbon Interactions at 31 GeV/c," Phys.Rev. C85 (2012) 035210.

[20] R. Snider, "Neutrino cross section measurements at MINERvA," in these proceedings (2012) .

[21] K2K Collaboration, A. Rodriguez et al., "Measurement of single charged pion production in the charged-current interactions of neutrinos in a 1.3-GeV wide band beam," Phys.Rev. D78 (2008) 032003.

[22] K2K Collaboration, C. Mariani et al., "Measurement of inclusive $\pi^{0}$ production in the Charged-Current Interactions of Neutrinos in a 1.3-GeV wide band beam," Phys.Rev. D83 (2011) 054023. 
[23] M. Ravonel, "Measurement of the muon neutrino flux and inclusive charged-current cross-section at T2K's near detector," in these proceedings (2012).

[24] G. Lopez, "Measurement of NC $\pi^{0}$ production and CC interactions using the ND280 P0D," in these proceedings (2012).

[25] H. Ray, "Recent Cross Section Measurements from MiniBooNE," in these proceedings (2012) .

[26] K. McFarland, “A review of neutrino cross-section measurements," Neutrino Conference Proceedings (2012) .

[27] T2K Collaboration, K. Abe et al., "Indication of Electron Neutrino Appearance from an Accelerator-produced Off-axis Muon Neutrino Beam,” Phys.Rev.Lett. 107 (2011) 041801.

[28] K. Sakashita, "Results from T2K," in these proceedings (2012) .

[29] G. Barr, "MINOS neutrino oscillation results," in these proceedings (2012) .

[30] MINOS Collaboration, P. Adamson et al., "Electron neutrino and antineutrino appearance in the full MINOS data sample,” Phys.Rev.Lett. (2013), arXiv:1301.4581 [hep-ex].

[31] M. De Serio, "Status of the OPERA search for muon-neutrino to tau-neutrino oscillations," in these proceedings (2012).

[32] R. Nichol, "Results from MINOS," Neutrino Conference Proceedings (2012) .

[33] T2K Collaboration, K. Abe et al., "First Muon-Neutrino Disappearance Study with an Off-Axis Beam," Phys.Rev. D85 (2012) 031103.

[34] Y. Itow, “Atmospheric Neutrinos: Results from running experiments," Neutrino Conference Proceedings (2012).

[35] J. Cao, "Reactor Neutrino Results," in these proceedings (2012) .

[36] LBNE Collaboration, “Conceptual design report,” LBNE-doc-5235-v9.

[37] A. Stahl, C. Wiebusch, A. Guler, M. Kamiscioglu, R. Sever, et al., "Expression of Interest for a very long baseline neutrino oscillation experiment (LBNO)," CERN-SPSC-2012-021, SPSC-EOI-007.

[38] K. Abe, T. Abe, H. Aihara, Y. Fukuda, Y. Hayato, et al., "Letter of Intent: The Hyper-Kamiokande Experiment — Detector Design and Physics Potential _," arXiv:1109.3262 [hep-ex].

[39] A. Badertscher, T. Hasegawa, T. Kobayashi, A. Marchionni, A. Meregaglia, et al., "A Possible Future Long Baseline Neutrino and Nucleon Decay Experiment with a 100 kton Liquid Argon TPC at Okinoshima using the J-PARC Neutrino Facility,” arXiv:0804.2111 [hep-ph] . 